ABSTRACT: Background: Among adults in the US, bipolar disorder affects $2.6 \%$ or 5.7 million individuals; $83 \%$ of cases are considered to be severe. Even when an accurate diagnosis of bipolar disorder is established, its treatment remains suboptimal, and those with the disorder often fail to receive any care or evidence-based care.

INTRODUCTION: A continuing medical education (CME)certified 25-item, multiple choice clinical practice assessment survey was developed to assess recognition and treatment of bipolar disorder, specifically, the use of LAIs in these patients.

METHODS: The survey included knowledge- and casebased multiple-choice questions completed confidentially online. The survey was launched on December 20, 2017 and hosted on the Medscape Education website. Participant responses were collected through January 31, 2018. Confidentiality was maintained, and responses were de-identified and aggregated before analyses.

RESULTS: ( $\mathrm{n}=1123$ psychiatrists; 305 primary care physicians [PCPs]):

- When asked about assessment tools in bipolar disorder, only $43 \%$ of psychiatrists and $36 \%$ of PCPs could identify the correct use of the MDQ screening instrument, while only $64 \%$ of psychiatrists and $51 \%$ of PCPs knew that the use of the MDQ can improve recognition of bipolar disorder in patients with depression;

- Psychiatrists were more likely to correctly identify the symptoms that most strongly support a diagnosis of bipolar disorder compared to PCPs $(76 \%$ vs $43 \%$, respectively);

- When asked about laboratory testing in mood disorders, $52 \%$ of psychiatrists and $46 \%$ of PCPs knew that laboratory testing can help exclude alternative causes for mood symptoms;

- The majority of both healthcare professionals $(73 \%$ $75 \%$ ) did not know that diagnosis of bipolar I disorder relies heavily on changes in activity, energy, and mood; - $87 \%$ of psychiatrists and $76 \%$ of PCPs did not identify oral aripiprazole as the only SGA not approved by the FDA for the maintenance treatment of bipolar I disorder;

- $49 \%$ of PCPs did not recognize lithium as the first choice for maintenance monotherapy for bipolar I disorder according to the guidelines;

- Only $19 \%$ of psychiatrists and $20 \%$ of PCPs correctly chose aripiprazole monohydrate and risperidone microspheres as the LAI SGAs indicated for use as monotherapy for patients with bipolar I disorder;

- When asked what is the most common barrier to prescribing LAI antipsychotics in patients with bipolar disorder, 34\% of psychiatrists selected "Patients fear of injectables"

CONCLUSIONS: This educational research identified psychiatrists and PCPs' current real-world clinical practices and gaps in the knowledge and competence in the diagnosis and assessment of bipolar disorder, and the treatment options for this condition. Further educational efforts tailored to address identified gaps for each audience are warranted.

Funding Acknowledgements: Otsuka

86

\section{Hyperfamiliarity for Unknown Faces in a Patient with Lewy Body Dementia}

Aghaegbulam Uga, $M D^{\prime}$; and Terngu Ibilah, $M D^{2}$

${ }^{1}$ Associate Professor and Program Director, Psychiatry and Internal Medicine Combined Program, Texas Tech University Health Science Center, El Paso, TX

${ }^{2}$ PGY 2 Resident, Psychiatry and Internal Medicine Combined Program, Texas Tech University Health Science Center, El Paso, TX

ABSTRACT: Title: "I know everybody here"

Hyperfamiliarity for unknown faces, a delusion of misidentification, is a rare disorder.

We present a 67-year-old female admitted with worsening cognitive impairment and poor self-care associated with parkinsonian symptoms of one-year duration. During evaluation, she was noted to relate to strangers with familiar gestures like people she already knew causing distress for family and care givers.

Workup revealed significant cognitive impairment, MOCA of $9 / 30$ and neuroimaging showing diffuse temporal lobe volume loss predominantly on the left.

Assessment was Lewy body dementia with hyperfamiliarity for unknown faces.

This rare presentation reflects the need for detailed examination and workup during evaluation.

87

\section{Efficacy Measures in an Open-label Dose- Optimization of an Amphetamine Extended- Release Oral Suspension in Children with Attention-Deficit/Hyperactivity Disorder}

Andrew Cutler, $\mathrm{MD}^{\prime}$; Antonio Pardo, $M D^{2}$; Thomas R. King, $M S, \mathrm{MPH}^{2}$; Judith C. Kando, PharmD, BCPP and and Barry K. Herman, $M D, M M M^{2}$

${ }^{1}$ Meridien Research, Bradenton, FL

2 Tris Pharma, Inc., Monmouth Junction, NJ 
ABSTRACT: Objectives: Report the efficacy of open-label amphetamine extended-release oral suspension (AMPH EROS) for the treatment of children with ADHD.

AMPH EROS has a 1-hr onset of effect and a duration of action of 13 hours and was approved by FDA for treatment of ADHD in children aged 6-17 years based on a doubleblind, placebo-controlled efficacy and safety study in children aged 6-12 years with ADHD. A significant treatment difference in change from pre-dose SKAMPcombined score was observed at the primary endpoint of 4 hours post-dose $(\mathrm{p}<0.0001)$ and each post-dose time point assessed $(1,2,4,6,8,10,12,13$ hours $)$.

Data reported here are from the 5-week, open-label dose optimization period. These efficacy data support the primary endpoint result.

METHODS: Males and females aged 6 to 12 years with ADHD enrolled and began open-label treatment with $2.5 \mathrm{mg}$ or $5 \mathrm{mg} /$ day of AMPH EROS titrated in $2.5-10 \mathrm{mg} /$ day increments until optimal dose (maximum $20 \mathrm{mg} /$ day). Doses could be decreased for tolerability. Subjects took morning AMPH EROS for 5 weeks. Other efficacy outcomes during the open-label dose optimization phase: ADHD-RS (ADHD-Rating Scale), CGI-S (Clinical Global Impression of Severity), CGI-I (CGI-of Improvement) and CPRS (Conners' Parent Rating Scale). All subjects were assessed for safety.

RESULTS: For the ITT population $(\mathrm{n}=99)$ : treatment with AMPH EROS was associated with a mean change in ADHD-RS-IV (baseline to end of the open-label dose optimization; week 6$)$ of $28.2( \pm 9.03)$ (Baseline score $=$ $41.3 \pm 7.95$ ). $90.9 \%$ of subjects had a change from baseline to open-label week 6 of $\geq 50 \%$ in the ADHDRS-IV total score and were defined as responders. The CGI-S scores decreased continuously from baseline, with a high 4.8 at baseline to a low of 2.0 at open-label week 6 . The percentage of subjects classified as moderately ill or greater correspondingly decreased from $97 \%$ at Baseline to $1 \%$ at open-label week 6 . The decrease in the CGI-I over the study was similar to the change in CGI-S scores. CPRS for most categories decreased continuously from Baseline to open-label week 6. Mean change from baseline to open-label week 6 on the CPRS inattention T-score subscale was $-25.3( \pm 14.38)$ and $-24.4( \pm 13.87)$. Adverse events $(>5 \%)$ reported during dose optimization were decreased appetite, insomnia, affect lability, upper abdominal pain, mood swings and headache.

CONCLUSION: AMPH EROS was effective in reducing symptoms of ADHD in this open-label dose optimization. The AE profile of AMPH EROS was consistent with those of other amphetamine products.

Funding Acknowledgements: This work was funded by Tris Pharma, Inc.
88

The Efficacy and Safety of Amphetamine Extended-Release Oral Suspension (AMPH EROS) in Children with Attention-Deficit/Hyperactivity Disorder

Judith C. Kando, PharmD, BCPP'; Thomas King, MS, $\mathrm{MPH}^{\prime}$; Antonio Pardo, $\mathrm{MD}^{\prime}$; and Barry K. Herman, $M D, M M M^{\prime}$

${ }^{1}$ Tris Pharma, Inc., Monmouth Junction, NJ

ABSTRACT: OBJECTIVES: To determine the efficacy and safety of amphetamine extended-release oral suspension (AMPH EROS) in the treatment of attentiondeficit/hyperactivity disorder (ADHD) compared with placebo in a dose-optimized, randomized, doubleblind study.

METHODS: The efficacy of AMPH EROS was evaluated in a laboratory classroom study conducted in 108 pediatric patients (aged 6-12 years) with ADHD. The study began with an open-label dose optimization (5 weeks) with an initial AMPH EROS dose of 2.5 or $5 \mathrm{mg}$ once daily in the morning. The dose could be titrated every 4-7 days in increments of $2.5-10 \mathrm{mg}$ until an optimal dose or the maximum dose of $20 \mathrm{mg}$ /day was reached. Subjects were required to tolerate a minimal dose of $10 \mathrm{mg} /$ day. Subjects then entered a 1-week randomized, double-blind treatment phase with the individually optimized dose or placebo. At the end of the week, raters evaluated the attention and behavior of the subjects in a laboratory classroom using the Swanson, Kotkin, Agler, M-Flynn, and Pelham (SKAMP-C) rating scale. SKAMP-C is a 13 -item teacher-rated scale that assesses manifestations of ADHD in a classroom setting.

The primary efficacy endpoint was change from pre-dose in the SKAMP-C score at 4 hours post dose. The key secondary endpoint efficacy parameters were onset and duration of clinical effect. The change scores from predose SKAMP-C scores at post dose time points $(1,2,6,8$, 10,12 and 13 hours) were used to evaluate the key secondary efficacy endpoints.

RESULTS: More boys (68.7\%) than girls participated in the study. The study population was $55.6 \%$ white, most patients had inattentive or combined type ADHD presentations. The primary efficacy endpoint, the change from pre-dose SKAMP-C score at 4 hours post dose was statistically significantly improved $(p<0.0001)$ compared with placebo. Each of the secondary efficacy endpoints were also significantly improved ( $p<0.0001$ at each time point) compared with placebo. Adverse events reported (frequency $>5 \%$ ) reported during the dose optimization 